\title{
Cleavage fracture modelling for RPV steels: Discrete model for collective behaviour of micro-cracks
}

DOI:

10.1115/PVP2013-97431

Link to publication record in Manchester Research Explorer

\section{Citation for published version (APA):}

Abu-Muharib, A., Jivkov, A. P., James, P., \& Yates, J. R. (2013). Cleavage fracture modelling for RPV steels:

Discrete model for collective behaviour of micro-cracks. In American Society of Mechanical Engineers, Pressure Vessels and Piping Division (Publication) PVP/ASME Pressure Vessels Piping Div. Publ. PVP (Vol. 6). American Society of Mechanical Engineers. https://doi.org/10.1115/PVP2013-97431

\section{Published in:}

American Society of Mechanical Engineers, Pressure Vessels and Piping Division (Publication) PVP|ASME Pressure Vessels Piping Div. Publ. PVP

\section{Citing this paper}

Please note that where the full-text provided on Manchester Research Explorer is the Author Accepted Manuscript or Proof version this may differ from the final Published version. If citing, it is advised that you check and use the publisher's definitive version.

\section{General rights}

Copyright and moral rights for the publications made accessible in the Research Explorer are retained by the authors and/or other copyright owners and it is a condition of accessing publications that users recognise and abide by the legal requirements associated with these rights.

\section{Takedown policy}

If you believe that this document breaches copyright please refer to the University of Manchester's Takedown Procedures [http://man.ac.uk/04Y6Bo] or contact uml.scholarlycommunications@manchester.ac.uk providing relevant details, so we can investigate your claim.

\section{OPEN ACCESS}




\section{CLEAVAGE FRACTURE MODELLING FOR RPV STEELS: DISCRETE MODEL FOR COLLECTIVE BEHAVIOUR OF MICRO-CRACKS}

\author{
Andrew Abu-Muharib \\ Modelling and Simulation Centre \\ The University of Manchester \\ Email: andrew.abu-muharib-2@postgrad.manchester.ac.uk
}

Peter James

AMEC

Risley, Warrington, UK

Email: Peter.James2@amec.com

\author{
Andrey P. Jivkov \\ Modelling and Simulation Centre \\ The University of Manchester \\ Email: andrey.jivkov@manchester.ac.uk
}

\author{
John R. Yates \\ Modelling and Simulation Centre \\ The University of Manchester \\ Email: john.r.yates@manchester.ac.uk
}

\begin{abstract}
The ability to predict variations in cleavage fracture toughness behaviour of ferritic RPV steels, accounting for the effects of irradiation and defect geometry, is vital to safety assessment and life extension decisions. Local approaches to cleavage fracture offer a promising methodology to accomplish such calculations. However, the limited progress achieved by improving the local failure probability expression suggests that the methodology for calculating global cleavage might not be adequately representing real material. The basis for the existing methodology is the weakest-link assumption that all individual failure events are independent and non-interacting. Here an approach is considered which utilises a microstructure-informed model incorporating the experimental knowledge needed to postulate deterministic criteria for particle rupture and micro-crack propagation, whilst accounting for the probabilistic distribution of particle sizes. This is then used in a lattice model that can help detail the evolution of the formation of micro-cracks on global failure, therefore inferring the suitability of the weakest-link assumption. Predicting the probability of cleavage fracture requires such models, as the macroscopic cleavage phenomenon is governed by a number of micro-structural features.
\end{abstract}

The material microstructure is represented by a regular lattice of truncated octahedral cells forming a computational sitebond model, with sites located at the cell centres and connected by two distinct sets of bonds. These bonds are modelled with structural beam elements, which represent all the possible relative deformations between coordinated sites. Particles of various sizes are distributed in the bonds, based on an experimentally determined distribution of cleavage initiating particles in RPV steel (Euro Material A). Although only elastic deformations are considered here, the results demonstrate that the interactions between individual failure events could potentially have a strong effect on the way global failure is reached.

Nucleation of micro-cracks by rupturing second-phase particles affects their subsequent formation. In particular, it was found that once formed there is a reduced probability of further development of micro-cracks at particles outside the crack planes and an enhanced probability of formation at particles along the crack planes. This will therefore influence the distribution of micro-crack sizes that could in principle be used to calculate the global probability of failure, and could lead to substantially different distributions of particle sizes, then used in the current local approach methods. 


\section{INTRODUCTION}

Cleavage fracture, a catastrophic failure mode that can occur in a range of structural materials, is caused by the rapid propagation of a micro-crack in the vicinity of a macroscopic flaw. It is now commonly accepted that, in ferritic steels, the origin of such micro-cracks is the rupturing of second-phase particles, predominantly carbides [1-4]. The particles present in steels differ in type, size and are distributed randomly in the volume [5]. These are typically elastic-brittle and, according to the current understanding, can rupture when overloaded by the plastically deforming matrix $[1,2]$. Hence, this limits the region where microcracks can form to the plastic zone ahead of the macroscopic flaw. The magnitudes and locations of the formed micro-cracks correspond to the sizes and positions of the initiating particles. Once the load on a component containing a macroscopic flaw increases, cleavage failure can occur given a critical combination of micro-crack size and matrix stress, and strain. This requires that a micro-crack of a specific size is formed or found at a spatial location where stresses are sufficient to cause rapid propagation. Reaching this critical combination does not preclude the formation of micro-cracks at reduced loads. The random distribution of particle sizes in the volume, and subsequent potential microcrack sizes, dictate the statistical nature of the cleavage fracture observed experimentally and reflected in the engineering Master Curve approach [6].

Computational modelling of cleavage processes must incorporate experimental knowledge and postulate deterministic criteria for particle rupture and micro-crack propagation, whilst accounting for the probabilistic distribution of particle sizes. Simulations can be performed with distributions of particle sizes at random spatial locations to derive a single fracture toughness value as the lower limit for given particle density. However, such a process is computationally demanding given the related plastic zone sizes involved in practical problems. Hence, existing models utilise a local approach methodology. The deterministic criteria for rupture and propagation are integrated into an expression for a critical particle (micro-crack) size in a given mechanical field. Thus, for a given load on a component containing a microscopic flaw, a critical size is defined for each location within the plastic zone ahead of the defect. This region is probabilistically distributed with an experimentally determined number density of particles. Experimental size distribution determines the probability of finding a particle larger than the given critical size. Hence, at each spatial location the model assigns a probability of local failure dependent on this predetermined critical size; relating directly to the local mechanical fields. Summation of all probabilities over the current plastic zone determines the global probability of failure.

Several local approach methods for assessing end-of-life fracture toughness of ferritic steels have been previously examined [7]. These approaches use different expressions to determine local probability of failure and a common methodology for accumulating such values into a global probability of failure. Updated expressions, to derive the individual probability of failure, account for the evolution in understanding over the last 25 years of the effects of stress, plastic strain and stress triaxiality on micro-crack formation and propagation. The existing methods assume that the individual failure events in a component are independent.

In all methods the summation of local probabilities over the plastic zone ahead of a macro-crack was based on the assumption that global failure is a weakest-link event. Existing methods fail to predict with sufficient accuracy and consistency the effects of temperature, irradiation and defect geometry on experimentally obtained fracture toughness values given common calculation parameters [7]. Furthermore, the methods are not able to predict the temperature dependence of fracture toughness in the ductile-to-brittle transition (DTBT) regime, despite disregarding constraint and irradiation effects [7].

A conceivable reason for these difficulties was that expressions for local probability of failure are functions of the local mechanical fields but not of the initiator size. This size dependence has been demonstrated experimentally and formed the first proposal for local probability as a function of both mechanical fields and initiator size [8]. A lack of size dependence in the approaches examined in [7] leads to significantly different predictions for the positions of critical initiators in a given stress field from those seen experimentally. Therefore, a new micromechanically based expression for calculating local probability of failure as a function of initiator size and local mechanical fields was developed in [7]. The new model yielded better predictions for the positions of critical initiators than the previous methods when compared to limited published experimental data [9]. When combined with the weakest-link statistics for global failure it produced an improvement in the predictions for temperature, irradiation and constraint effects on fracture toughness $[10,11]$. It is however noted that under higher levels of plasticity, particularly at lower constraints, the model predictions for cleavage probability are still unsatisfactory. It is also under such conditions that an increased distribution of micro-cracks is more likely and the suitable use of the weakest-link summation less clear.

Work is presented to support the conjecture that defect interactions could have substantial effect on cleavage fracture calculations. The results presented are not able to explicitly show that global failure is not a weakest-link event. Rather, they demonstrate that interaction effects have the potential to alter the methodology for calculating global failure probability from the exiting weakest-link assumption. Although, this implicitly invalidates the weakest-link theory, it can still be used as a valid approximation when interactions are negligible, e.g. at low plastic strains. At increased plastic strains, fracture toughness predictions begin to deviate from the Master Curve, rendering the weakest-link theory deficient 
in predicting global failure, such as with temperature changes.

\section{MODEL METHODOLOGY}

The material studied is an RPV 22NiMoCr37 ferritic steel known as Euro Material A, also used as the subject case in [7], for which we have the mechanical and fracture toughness properties. The model is based on an experimentally determined density and distribution of cleavage initiating particles in Material A [12]. Metallographic examination of provided a density of particles, $\rho=7.6 \times 10^{17} \mathrm{~m}^{-3}$. Furthermore, it provides a probability of particle sizes which was incorporated within a Weibull-type statistical analysis Eqn. (1); with $\beta=2.7$ being the shape parameter, and $r_{o}=0.036 \mu \mathrm{m}$ being the scale parameter representing the mean particle size.

$$
f(r)=\frac{\beta}{r_{o}}\left(\frac{r}{r_{0}}\right)^{-\beta-1} \exp \left[-\left(\frac{r}{r_{0}}\right)^{-\beta}\right]
$$

The expression for the probability of particle failure is based on experimental observations that demonstrate this probability depends not only on the mechanical fields but also on the particle size, in a similar methodology to that adopted by the WST model [13]. The rationale is that larger particles have a higher probability of failure than smaller particles under equal mechanical conditions. Hence, Eqn. (1) is pertinent in determining particle size and distribution data which forms the bases of the lattice model failure criterion. This advances previous models used to describe the mechanism of micro-crack formation [9]. The particles are assumed to be elastic-brittle with elastic constants equal to those of the matrix as a first approximation.

The material was modelled at the meso-scale; the length scale most relevant to the mechanism of microscopic failure or micro-crack formation. This mechanism is associated with the presence of particles that behave as failure initiators within the material. A physical region of the material ahead of a crack tip can be structured into cells, such that particles (failure initiators) are distributed at the boundaries between the cells. We sought to construct a regular model of the microstructure based on previous works; carefully selecting the correct cell shape and orientation which suitably represents real materials. Monte Carlo studies relating to properties of three-dimensional Poisson-Voronoi tessellations of space, provide insight into the shape selection [14]. According to these studies, the average cell shape and coordination is closest to the truncated octahedron, which can fill the space compactly and regularly. This solid is bounded by six squares and eight regular hexagons, Fig. (1). Subsequently this unit cell is used to construct a computational site-bond model.
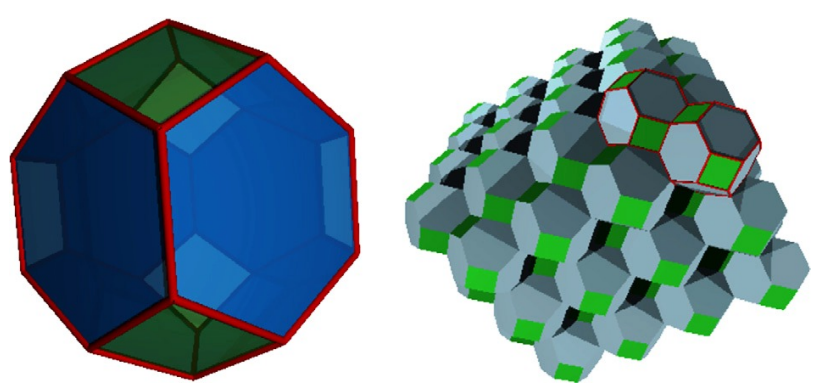

FIGURE 1. UNIT CELL (TRUNCATED OCTAHEDRON) AND 3D REGULAR CELLULAR ASSEMBLY REPRESENTING MATERIAL MICROSTRUCTURE [15].

The sites from each set are connected by bonds to the neighbouring six sites of the same set and to the adjoining eight sites of the other set, Fig. (2).

The site-bond model is constructed using two types of bond, denoted $B_{1}$ and $B_{2}$, with lengths $L_{1}=L$ and $L_{2}=\sqrt{3} L / 2$, respectively, where $L$ is the unit cell size, i.e. see Fig. (2). The bonds are modelled as structural beam elements, assuming local isotropy, with circular cross-sections and two distinct radii $R_{1}$ and $R_{2}$ respectively. It is possible to alter the stiffness coefficients of each type of bond independently, making the model sufficiently variable to represent any predefined macroscopic elastic behaviour [15]. Thus the stiffness coefficients of the two types of bonds are dependent on the ratios $R_{1} / L, R_{2} / L$ and $G_{b} / E_{b}$, where $E_{b}$ is the modulus of elasticity and $G_{b}$ the modulus of rigidity of the bonds. As discussed, the material selected for analysis is a ferritic steel. Typical values for the macroscopic elastic properties are modulus of elasticity $E=200 \mathrm{GPa}$ and Poissons ratio $v=0.3$. The linear elastic behaviour of this material is represented by the site-bond model with the following bond properties in Tab. (1). Further details of this modelling methodology and sitebond tuning can be found in [15].

TABLE 1. TABLE OF ASSOCIATED BOND PROPERTIES FOR SITE-BOND MODEL.

\begin{tabular}{|c|c|c|c|c|}
\hline$R_{1} / L$ & $R_{2} / L$ & $G_{b} / E_{b}$ & $v$ & $E_{b}(M P a)$ \\
\hline 0.1 & 0.16 & 0.33 & 0.5 & 1936483 \\
\hline
\end{tabular}

The model is currently developed to represent the elastic behaviour of the material. Thus, upon loading, the sites experience the forces from the linearly elastic responses of adjacent bonds. Sites have six independent degrees of freedom: three translational and three rotational. Correspondingly, the bonds are capable of transferring axial and shear forces, and torsion and bending moments. A particle positioned in a bond is allowed to fail either 


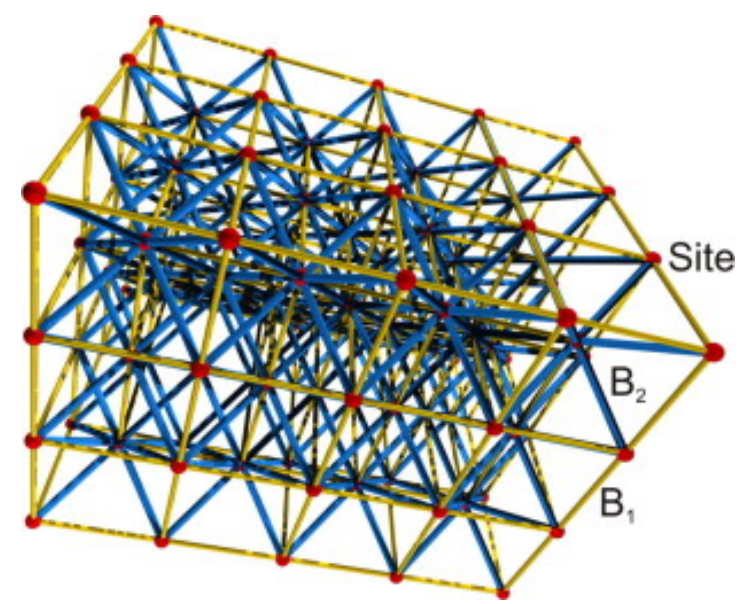

FIGURE 2. SITE-BOND ASSEMBLY OF A 3D SOLID-PHASE MODEL [15].

in tension, forming a Mode I micro-crack, or in shear, forming a Mode II micro-crack. The criterion for particle failure is based on a critical value of the strain energy density associated with either opening or sliding penny-shaped cracks. These strain energy densities are given respectively in Eqn.(2) and Eqn.(3) [16]

$$
\Delta E_{I}=\frac{4(1-v)}{3 \mu} \sigma_{n}^{2} r^{3}
$$

and

$$
\Delta E_{I I}=\frac{8(1-v)}{3(2-v) \mu} \sigma_{t}^{2} r^{3}
$$

where $\mu$ and $v$ are the shear modulus and Poisson's ratio, $r$ is the particle size and $\sigma_{n}, \sigma_{t}$ are the normal and shear stresses acting on the particle from a given bond. Hence, it is assumed that a crack may form if one of the expressions Eqn.(2) and (3) reaches a critical value for a given combination of crack sizes and stresses.

The model used for the simulation reported here occupies the region $(10 L, 10 L, 10 L)$ with respect to a coordinate system $\left(X_{1}, X_{2}, X_{3}\right)$ orientated along the principal axes of the cell. This provides the model with 1729 individual particles that can be strained and critical bonds subsequently removed from the system following failure. It should be noted that this arrangement is surrounded by a framework through which prescribed displacements are applied to the model; the bonds of this surrounding are not removable.

The model behaviour is purely elastic and so magnitudes of the applied loads can be treated as arbitrary. Once a load is applied, the strain energy densities in all particles associated with their sizes and the stresses from the bonds are placed in descending order. The combination of a particle and a bond that provide the largest potential for energy release upon cracking gives the critical bond in the system. This bond is then removed from the system and a new solution is obtained. The new result can begin from the same external load as previously described due to the assumed elastic behaviour. Thus the evolution of damage (micro-cracks) in the system is found as a sequence of equilibrium solutions with consecutively removed bonds one per simulation step.

At each simulation step, $i$, the model is subjected to fixed macroscopic strains along one or more of the axis, $\varepsilon_{m}$ via prescribed displacements. The corresponding macroscopic stresses, $\sigma_{m}^{i}$, are determined from the reaction forces. These macroscopic stresses vary with the accumulation of micro-cracks, producing changes in the elastic modulus of the material. Such alterations are characterised by a damage parameter, $D^{i}=1-E^{i} / E$, where $E=\sigma^{0} / \varepsilon^{0}=200 \mathrm{GPa}$ is the initial elastic modulus of the material and $E^{i}=\sigma^{i} / \varepsilon^{i}$, is the current modulus. Thus, the sequence of elastic solutions performed at arbitrary magnitude of the external load provides an evolution of an important relation between mechanical properties of the damage in the system measured as the relative change of the modulus of elasticity.

The real stress-strain curve, corresponding to the failure events at the simulation steps, can be obtained upon prescribing a particular critical value for the strain energy density associated with crack formation. This determines the macroscopic stress and strain at which a particular failure event has occurred. Hence, the results from the simulation steps in terms of bond stresses are used to 'cut-back' the arbitrary applied load to the point of failure for each step. The macroscopic stress-strain behaviour of the material is therefore represented by the sequence $\varepsilon^{i}, \sigma^{i}$ of cut-back stresses and strains. Consequently, the current version of the model considers only elastic behaviour. Therefore the effects of temperature or irradiation on the collective behaviour of micro-cracks could not be studied. These effect$\mathrm{s}$ are predominantly mediated by changes in the yield and flow properties, which are not currently implemented. However, an approach to include plasticity is under development and will be reported in a future publication.

\section{RESULTS}

Four separate cases of varying macroscopic crack tip constraint were considered with corresponding T-stress values. Tab. (2) provides a summary of the cases studied. These Tstress values represent analyses undertaken on three-point bend specimens [17]. Negative (compressive) values serve to reduce the crack-tip constraint, increasing the apparent fracture toughness. The corresponding T-stress values were implemented into the model by introducing displacements, $u_{1}, u_{2}$, in the principal directions, $X_{1}, X_{2}$, respectively. A number of aspects were considered, including analysis of the mechanical characteristics of the model under loading, damage onset and probability of failure 
TABLE 2. TABLE OF RELATIVE STRAINS AND DISPLACEMENTS FOR EACH OF THE T-STRESS CASES CONSIDERED [17].

\begin{tabular}{|c|c|c|c|c|}
\hline Case & 1 & 2 & 3 & 4 \\
\hline T-Stress $(T / \sigma=0.0)$ & 0.0 & -0.5 & -1.0 & -1.5 \\
\hline$\varepsilon_{1} / \varepsilon$ & 2.625 & 1.75 & 0.875 & 0 \\
\hline$\varepsilon_{2} / \varepsilon$ & 2.625 & 3 & 3.375 & 3.75 \\
\hline$u_{1}$ & 1 & 0.583 & 0.259 & 0 \\
\hline$u_{2}$ & 1 & 1 & 1 & 1 \\
\hline
\end{tabular}

for both individual and collective behaviour.

The relationship between the onset of macroscopic damage and stress $\sigma_{22}$ or strain $\varepsilon_{22}$ is predicted by the model and shown in Figs. (3) and (4), respectively. The macroscopic damage parameter, $D$, is shown to increase in both plots for decreasing constraint values of $T / \sigma$. However, the peak stress $\sigma_{22}$ in the principal loading direction for the most constrained case, $T / \sigma=0.0$, is significantly larger than the least constrained case, $T / \sigma=-1.5$. A similar trend can be seen when comparing the initial constrained case with the remaining negative T-stress cases, such that there is a marked increase in macroscopic damage and a significant reduction of the main applied strain, $\varepsilon_{22}$. Cases 2-4 show similar features, with increasing values for maximum damage.

Since the comparison is made for equivalent number of failure events in all loading cases, the reduced constraint is equivalent to increasing the damage required to reach this number of failures. Assuming this value produces global failure of the assembly, the reduced constraint cases need more damage to be produced prior to failure, which will occur at lower principal stress and higher principal strain. This interpretation demonstrates the expected behaviour of cracked geometries with various constraints. The results presented, show the model was capable of reproducing the expected mechanical behaviour of the material in the elastic regime and at different constraint conditions.

Given the simplicity of the model; utilising only the elastic modulus and Poissons ratio to calibrate the elastic properties of the bonds and an experimental particle size distribution for this material to obtain distributions of failure strengths of these bonds, the results obtained are promising. The development of damage is found to be exclusively due to Mode I micro-cracks emerging normal or inclined to the applied load. The linear behaviour prior to failure is reproduced, and non-linearity increases with the progression of damage. Simulations with different surface energies were performed. These demonstrated that model is also able to predict the increase in work needed to overcome particles of higher surface energies. Although there is a noticeable difference in the applied strain $\varepsilon_{22}$ between $T / \sigma=0.0$ and

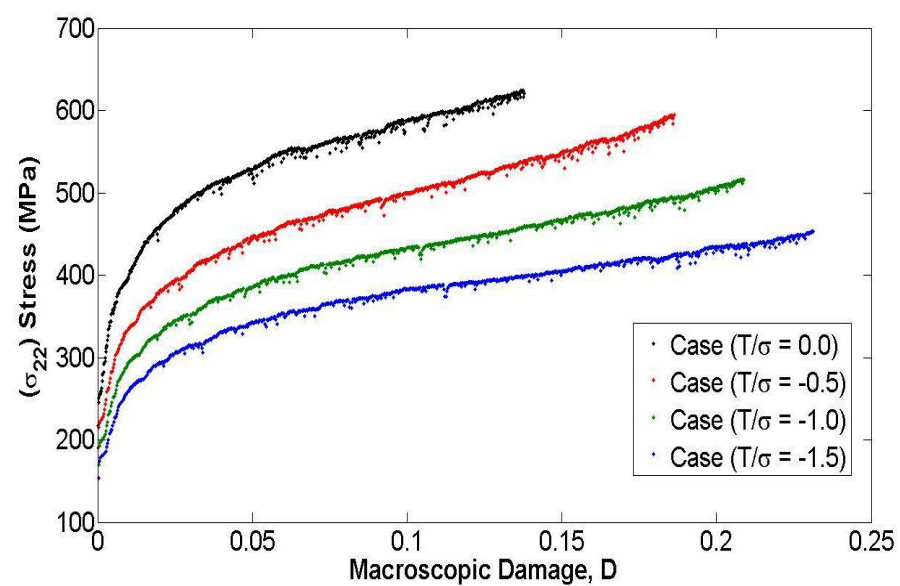

FIGURE 3. RELATIONSHIP BETWEEN STRESS $\sigma_{22}$ AND MACROSCOPIC DAMAGE FOR THE FOUR T-STRESS CASES.

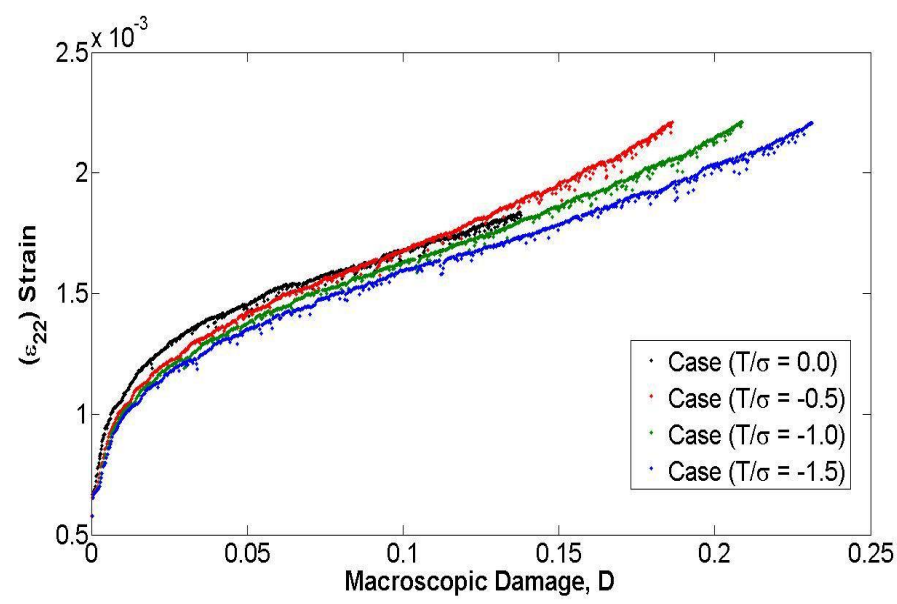

FIGURE 4. RELATIONSHIP BETWEEN STRAIN $\varepsilon_{22}$ AND MACROSCOPIC DAMAGE FOR THE FOUR T-STRESS CASES.

the other three cases, there is little change in maximum applied strain between the negative T-stress cases. However, a marked change in the applied stress $\sigma_{22}$ can be observed, demonstrating that there is adequate variation between the cases for further study of the model.

For the current model, there is no simple method to define the point of global failure for the assembly. Such a point will depend on the entire structure of accumulated damage, including the formed isolated or linked micro-cracks. Presently, a simplified study to infer the interaction effects between micro-cracks can be considered. Each of the particles in the assembly is attached a local probability of failure according to its size and the stresses acting on the particle in the undamaged state of the assembly. Note that these individual probabilities of failure could be exploited if a local approach were applied to calculate global 


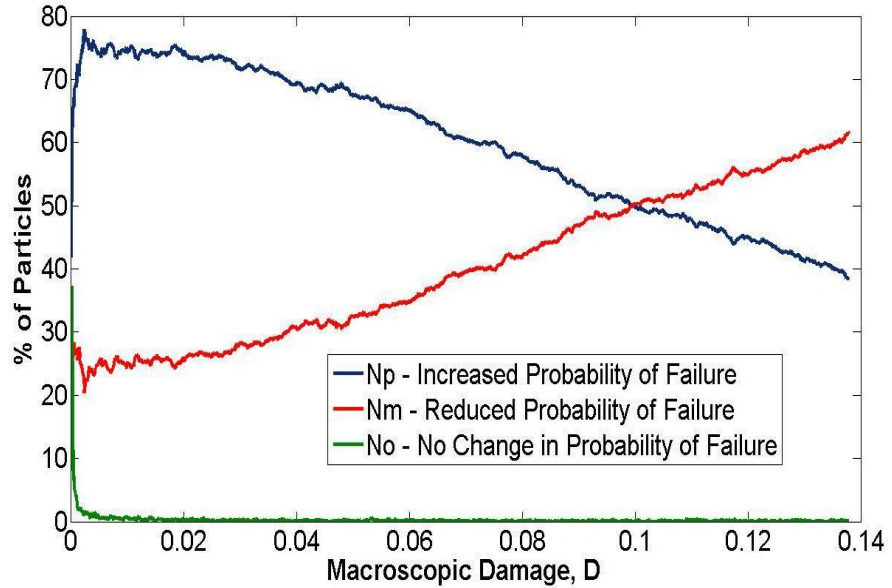

FIGURE 5. CHANGES IN PERCENTAGE PROBABILITY OF PARTICLE FAILURE WITH DAMAGE FOR $T / \sigma=0.0$.

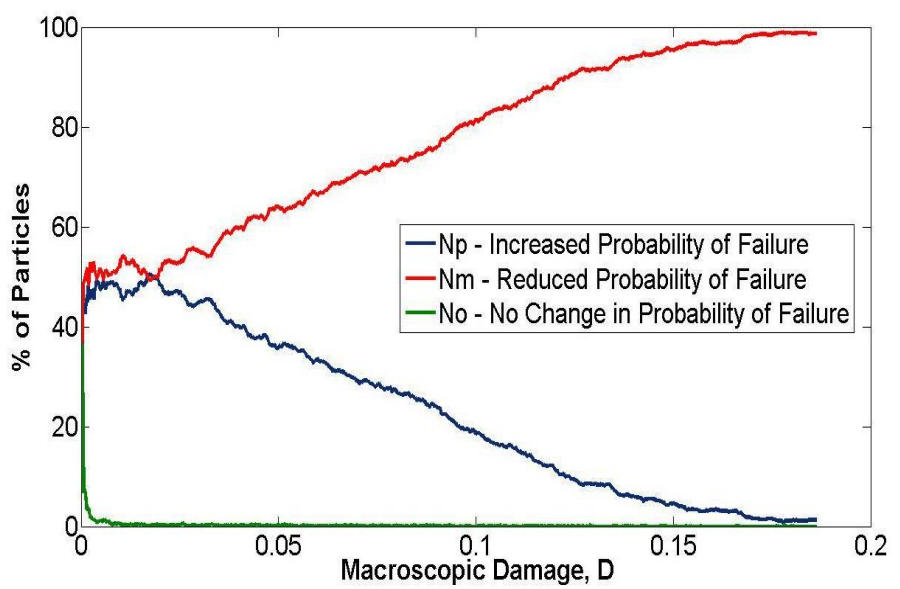

FIGURE 6. CHANGES IN PERCENTAGE PROBABILITY OF PARTICLE FAILURE WITH DAMAGE FOR $T / \sigma=-0.5$.

probability of failure. In the model employed, after each failure event the probabilities of rupture of all remaining particles can be recalculated using the new redistributed stresses and strains. This has been performed for the results with the four cases studied and several important observations are reported below. Results relate the percentage of particles with increased, reduced and unchanged probability as a function of macroscopic damage. Maximum percentage increase and reduction in failure probability, are also considered, with the onset of macroscopic damage.

Figs. (5) to (8) show the percentage of particles in the model $\left(N_{p}, N_{m}, N_{o}\right)$ which have increased, decreased or seen no change in the probability of failure, respectively, in relation to the corresponding macroscopic damage parameter, $D$, for the individual T-stress cases. There is a marked reduction in the number of particles which show an increased probability of failure as the model

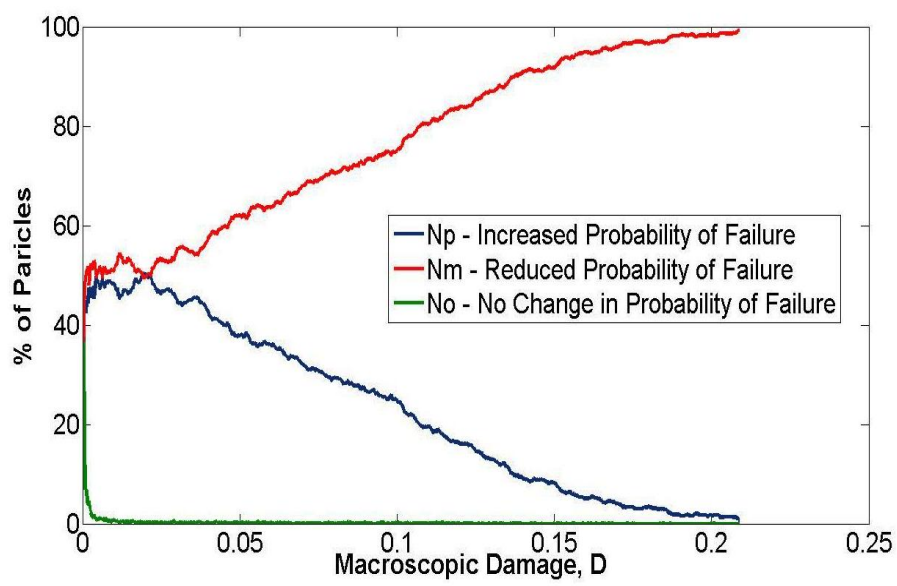

FIGURE 7. CHANGES IN PERCENTAGE PROBABILITY OF PARTICLE FAILURE WITH DAMAGE FOR $T / \sigma=-1.0$.

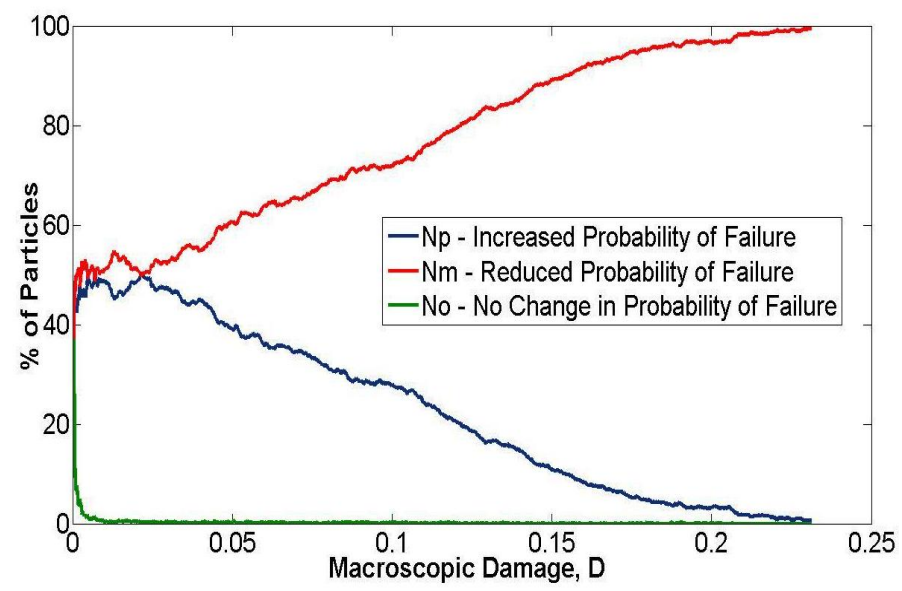

FIGURE 8. CHANGES IN PERCENTAGE PROBABILITY OF PARTICLE FAILURE WITH DAMAGE FOR $T / \sigma=-1.5$.

becomes continuously damaged; suggesting that increased damage (formation of micro-cracks) produces a reduction in bond failure. This is confounded by the increase in the number of particles with reduced probability of failure within the model, given the onset of macroscopic damage. Furthermore, aside from the initial stages of damage evolution, there are few particles with unchanged probability of failure. The number of these is practically zero for $D>0.01$. Hence, even at low levels damage and the subsequent formation of micro-cracks has direct effect upon all particles in the system.

The T-stress cases, $T / \sigma=-0.5,-1.0,-1.5$, show identical trends but with differing final microscopic damage values associated with $50 \%$ of failed particles. However, the $T / \sigma=0.0$ case does not display the same characteristics. The point at which increased and decreased probability percentage failure of particles 


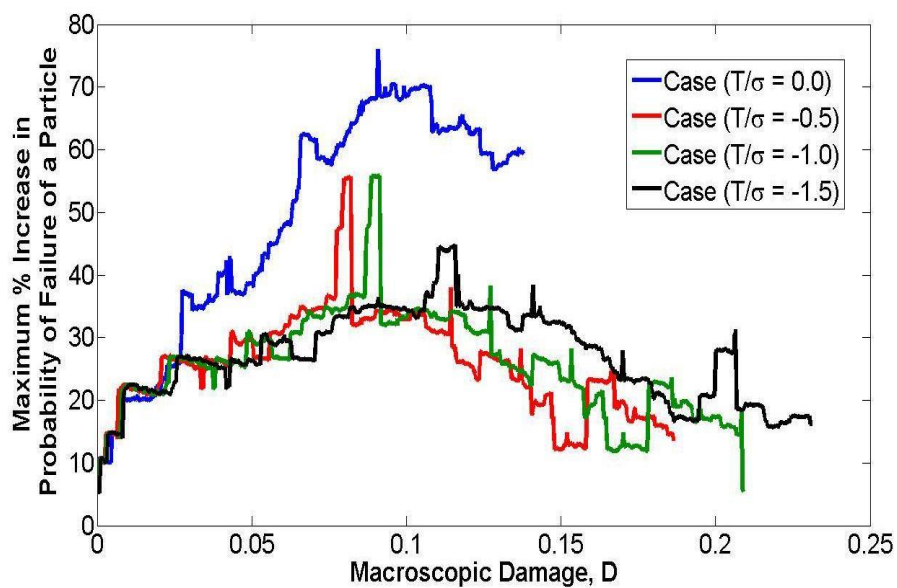

FIGURE 9. COMPARISON OF THE MAXIMUM PERCENTAGE INCREASE OF PROBABILITY OF PARTICLE FAILURE FOR THE FOUR T-STRESS CASES.

intersects is at a higher macroscopic damage level, than that of the other three cases. Suggesting, that under lower constraint the effect of macroscopic damage has a greater significance on the probability of failure of bonds within the model. However, the effect upon probability of particle failure plateaus as $N_{p} \rightarrow 0 \%$ and $N_{m} \rightarrow 100 \%$, before the maximum damage parameter is met.

A more succinct comparison of the effect of both damage and T-stress upon probability of particle failure can be seen in Figs. (9) and (10). Fig. (9) shows the greatest increase of probability of particle failure for each of the T-stress cases. The most constrained case, $T / \sigma=0.0$, has distinct characteristics when compared to the other three cases. Probability of increase in particle failure reaches a peak of around $80 \%$ with the onset of damage. Cases with lower constraint show similar trends with respect to the increase of failure probability with a maximum at around $40 \%$. The presence of a peak in the increase of failure probability at approximately the same global damage for all cases suggests that this condition might be used in the future to define the point of global failure probability. The reduction in the apparent maximum failure probability after the peak suggest$\mathrm{s}$ that the microstructure has exhausted its resistance potential.

Fig. (10) shows the greatest reduction in the probability of particle failure for each of the T-stress cases. There is a distinc$\mathrm{t}$ trend of an increasing reduction of particle failure probability with reduced constraint. The most noticeable difference comes from a direct comparison between the three negative T-stress cases and that of the highest constraint. Clearly lowering the constraint leads to increased reduction in the probability of failure, which could reach around - 80-90\%.

The results presented support the conjecture that the interaction between micro-cracks could have a significant effect on the approach to global failure. It was observed, but not shown

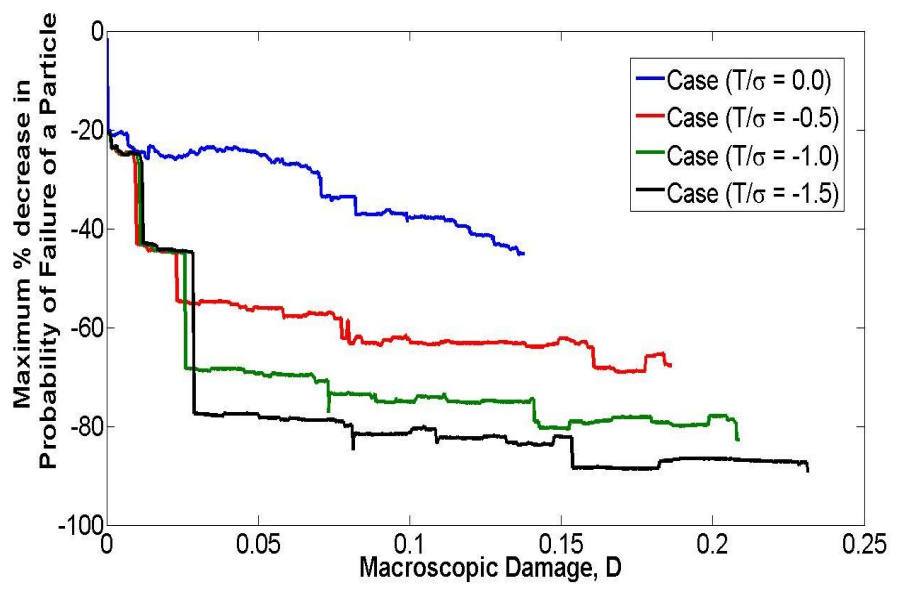

FIGURE 10. COMPARISON OF THE MAXIMUM PERCENTAGE REDUCTION OF PROBABILITY OF PARTICLE FAILURE FOR THE FOUR T-STRESS CASES.

graphically here, that the maximum increases in the failure probabilities were always found at particles situated next to and on the plane of previously formed micro-cracks. While maximum reductions in the failure probabilities were found at particles situated next to but normally inclined to previously formed microcracks on parallel planes.

\section{DISCUSSION}

Predicting the probability of cleavage fracture requires microstructure-informed models, as the macroscopic phenomenon is governed by a number of microstructural features; such as grain sizes and orientations and second-phase particle sizes and distributions. Engineering models consider the material as a continuum and must define an expression for individual failure probability at a material point and a methodology for calculating the probability of cleavage from the individual probabilities in a continuum. The microstructural information required in continuum models is therefore: (i) the probability density of particle sizes to provide individual failure probabilities dependent on local mechanical fields; and (ii) the number density of particles to provide scaling for the global probability of failure.

The limited progress achieved by improving the local failure probability expression suggested that the methodology for calculating global cleavage might not be representing the material response adequately. The basis for the existing methodology is the weakest-link assumption that all individual failure events are independent and non-interacting. As a result, the following scenario is the foundation of current methodology:

- For given constant stress and strain tensors within a material volume the criteria for particle rupture and micro-crack propagation in a particular local approach model will deter- 
mine a critical particle size.

- The probability density of particle sizes will then provide a local probability of failure as the cumulative probability of finding a particle of larger size.

- Size of the material volume and the number density of particles determine the sum of particles larger than the critical size within the volume.

- As interactions are not considered, all of these particles contribute to the summation over the volume.

- Thus potentially, all particles larger than the critical size found in the volume are considered as micro-cracks with equal failure probability.

This scenario corresponds to a typical calculation based on finite element analysis, where the material volume with constant stresses and strains is either the whole finite element, for elements with linear interpolation, or the volume of one integration point, for elements with higher-order interpolation. More importantly, the same process is then repeated for summing up the elemental failure probability over the plastic zone to obtain the global probability of failure. At this level the failures of elements are again considered as independent events.

Although the proposed model still assumes elastic behaviour, the proper mechanical response of the system could be reproduced under several cases with variable constraint. This includes stress-strain non-linearity emerging solely from the formation and interaction of micro-cracks in the system and the development of macroscopic damage. It was shown that lower constraint cases require increasingly larger damage to be produced in order to reach global assembly failure, which would occur at increasingly higher remote strains and progressively lower remote stresses. Moreover, the nucleation of micro-cracks in the system affected practically all particles remaining intact, by altering their probability of failure. After little damage, the number of particles with unchanged failure probability reduced to zero. Whilst the remaining set of particles experienced either increased or reduced probability of failure.

The increase of maximum particle failure probability was most pronounced at highest constraint and decreased with a corresponding reduction in constraint. Thus, particles could experience up to $80 \%$ increase of failure probability at high constraint and up to $40-50 \%$ at lower constraint. This correlates well with the experimental knowledge for the constraint effect. The opposite was seen for the maximum decrease of the particle failure probability. In this case, the effect of constraint was most pronounced, as the lowest constrained case considered showed up to $90 \%$ reduction in the probability of failure of some particles. Once again the expected behaviour at low constraint condition$\mathrm{s}$ is encountered, where larger loads are needed to cause global failure.

\section{SUMMARY}

In summary, the results from the discrete micro-mechanical model, even at this early stage of development with elastic behaviour and simple failure criterion, provided support for the conjecture that micro-crack interaction effects need to be accounted for in a future methodology for predicting cleavage fracture toughness.

This work demonstrated that the nucleation of microcracks by rupturing second-phase particles affects the subsequent micro-crack formation. In particular, formed micro-cracks reduce the probability of formation of micro-cracks at particles outside the crack planes and enhance the probability of formation of micro-cracks at particles along the crack planes. This affects the distribution of micro-cracks sizes that should in principle be used to calculate the global probability of failure. The microcracks could be substantially different from the distribution of particle sizes used in the current local approach methods. Strictly, these observations are valid for the elastic-brittle behaviour of beams used in the study. Further investigation with elasticplastic material model will be carried out to support them. It is not clear at present, whether the interaction effects can be explicitly accounted for in an improved expression for local failure probability or in a novel methodology for global probability calculations. More experimental and modelling work is needed to gain a better understanding of these effects and possibly derive an advanced local approach method.

The discrete lattice model used in this work offers an advantageous way of modelling distributed damage, i.e. the behaviour of a population of micro-cracks. However, the model needs to be further advanced and there are a number of points that need to be considered in this development. Firstly, the behaviour of the bonds needs to be elastic-plastic, which will allow studies of materials with different yield and hardening behaviour, in particular ferritic steels at different temperature and irradiation regimes. Secondly, the criterion for particle failure/micro-crack formation needs to be based on the strain and stress tensors at sites representing particles. This can be deduced from all forces in the adjacent bonds. Currently the failure is considered as being induced by each of these bonds in isolation. Thirdly, a criterion for global failure needs to be postulated as a function of the structure of the micro-cracks population. Nonetheless, the model shows an ability to provide further insight to the local mechanisms in the DTBT regime.

\section{REFERENCES}

[1] McMahon, C., and Cohen, M., 1965. "Initiation of cleavage in polycrystalline iron”. Acta Metallurgica, 13(6), pp. 591604.

[2] Gurland, J., 1972. "Observations on the fracture of cementite particles in a spheroidized $1.05 \% \mathrm{C}$ steel deformed at 
room temperature". Acta Metallurgica, 20(5), pp. 735741.

[3] Hahn, G., 1984. "The influence of microstructure on brittle fracture toughness". Metallurgical and Materials Transactions A, 15(6), pp. 947-959.

[4] Lee, S., Kim, S., Hwang, B., Lee, B., and Lee, C., 2002. "Effect of carbide distribution on the fracture toughness in the transition temperature region of an SA 508 steel". Acta materialia, 50(19), pp. 4755-4762.

[5] Kroon, M., and Faleskog, J., 2005. "Micromechanics of cleavage fracture initiation in ferritic steels by carbide cracking". Journal of the Mechanics and Physics of Solids, 53(1), pp. 171-196.

[6] Wallin, K., 2010. "Structural integrity assessment aspects of the Master Curve methodology". Engineering Fracture Mechanics, 77(2), pp. 285-292.

[7] Jivkov, A. P., Lidbury, D. P. G., and James, P., 2011. "Assessment of Local Approach Methods for Predicting Endof-Life Toughness of RPV Steels". ASME Conference Proceedings, 2011(44564), pp. 293-302.

[8] Wallin, K., Saario, T., and Törrönen, K., 1986. "Fracture of brittle particles in a ductile matrix". International journal of fracture, 32(3), pp. 201-209.

[9] Hohe, J., Friedmann, V., Wenck, J., and Siegele, D., 2008. "Assessment of the role of micro defect nucleation in probabilistic modeling of cleavage fracture". Engineering fracture mechanics, 75(11), pp. 3306-3327.

[10] Jivkov, A. P., and James, P., 2013. "Cleavage modelling with experimental particle size distribution and novel particle failure criterion". In 13th International Conference on Fracture.

[11] James, P., Ford, M., and Jivkov, A. P., 2013. "Predictions of fracture toughness for weld material when adopting a novel particle failure criterion and a measured, experimental particle distribution". In ASME Conference Proceedings, Vol. 2013, ASME.

[12] Ortner, S., Duff, J., and Beardsmore, D., 2005. Characterisation of Euro A reference steel for application of EOH model of brittle fracture. Tech. rep., SERCO Assurance. in: Technical Report SA/EIG/15234/R003 Project PERFECT.

[13] Wallin, K., Saario, T., and Törrönen, K., 1984. "Statistical model for carbide induced brittle fracture in steel". Metal Science, 18(1), pp. 13-16.

[14] Kumar, S., Kurtz, S., Banavar, J., and Sharma, M., 1992. "Properties of a three-dimensional Poisson-Voronoi tesselation: A Monte Carlo study". Journal of Statistical Physics, 67(3), pp. 523-551.

[15] Jivkov, A., and Yates, J., 2012. "Elastic behaviour of a regular lattice for meso-scale modelling of solids". International Journal of Solids and Structures, 49, pp. 3089-3099.

[16] Sneddon, I., 1969. "Transform solutions of crack problem$\mathrm{s}$ in the theory of elasticity". ZAMM-Journal of Applied
Mathematics and Mechanics/Zeitschrift für Angewandte Mathematik und Mechanik, 49(1-2), pp. 15-23.

[17] Sherry, A. H., France, C. C., and Goldthorpe, M. R., 1995. "Compendium of T-Stress solutions for two and three dimensional cracked geometries". Fatigue \& Fracture of Engineering Materials \& Structures, 18(1), pp. 141-155. 